\title{
Mechanical Properties of Single Muscle Fibers: Understanding Poor Muscle Quality in Older Adults with Diabetes
}

\author{
Eun-Jeong Lee ${ }^{1,2}$, Hak Chul Jang ${ }^{3}$, Kyung-Hoi Koo ${ }^{4}$, Hye-Young Kim ${ }^{5}$, Jae-Young Lim ${ }^{2}$ \\ ${ }^{1}$ Department of Kinesiology, School of Health and Human Science, Concordia University Irvine, Irvine, CA, USA \\ ${ }^{2}$ Department of Rehabilitation Medicine, Seoul National University Bundang Hospital, Seoul National University College of Medicine, Seongnam, Korea \\ ${ }^{3}$ Department of Internal Medicine, Seoul National University Bundang Hospital, Seoul National University College of Medicine, Seongnam, Korea \\ ${ }^{4}$ Department of Orthopedic Surgery, Seoul National University Bundang Hospital, Seoul National University College of Medicine, Seongnam, Korea \\ ${ }^{5}$ Division of Liberal Arts and Science, Korea National Sport University, Seoul, Korea
}

\section{Corresponding Author:}

Jae-Young Lim, MD, $\mathrm{PhD}$

Department of Rehabilitation

Medicine, Seoul National University

Bundang Hospital, Seoul National

University College of Medicine, 82

Gumi-ro 173beon-gil, Bundang-gu,

Seongnam, 13620, Korea

E-mail: drlim1@snu.ac.kr

ORCID:

https://orcid.org/0000-0002-9713-5416

Received: November 12, 2020

Revised: December 20, 2020

Accepted: December 21, 2020
Background: While aging causes muscle weakness, type 2 diabetes mellitus (T2DM) is also considered a high-risk factor for the induction of skeletal muscle weakness. Previous studies have reported increased collagen content in insulin-resistant skeletal muscles. Here, we studied the mechanical properties of aged skeletal muscle in patients with T2DM to investigate whether aged skeletal muscles with T2DM induce higher passive tension due to the abundance of extracellular matrix (ECM) inside or outside of the muscle fibers. Methods: Samples from the gluteus maximus muscles of older adults with diabetes (T2DM) and non-diabetic (non-DM) older adults who underwent elective orthopedic surgery were collected. Permeabilized single muscle fibers from these samples were used to identify their mechanical properties. Sodium dodecyl sulfate-polyacrylamide gel electrophoresis (SDS-PAGE) was used to quantify titin and fiber type distributions in these samples. Results: We confirmed a significant predominance of type I fiber ratio in both T2DM and non-DM aged muscles. While the average cross-sectional area and maximal active tension of the single fibers were smaller in the T2DM group than those in the non-DM group, the difference was not statistically significant. T2DM subjects showed significantly greater passive tension and lower titin-/ECM-based passive tension ratios than those in non-DM subjects, which indicated that more ECM but less titin contributed to the total passive tension. Conclusion: Based on our findings, we concluded that T2DM may cause increased passive stiffness of single skeletal muscle fibers in older adults because of an excessive accumulation of ECM in and around single muscle fibers due to increased insulin resistance.

Key Words: Diabetes mellitus, Older adults, Skeletal muscle, Passive tension, Titin

\section{INTRODUCTION}

Type 2 diabetes mellitus (T2DM) is the most widespread metabolic disorder worldwide, with a continuously increasing prevalence throughout the last decade. ${ }^{1,2)}$ Khan et al. ${ }^{3)}$ demonstrated that an estimated 462 million individuals worldwide were affected by T2DM in 2017 , corresponding to $6.28 \%$ of the world's population. In high-income countries, T2DM affects about $70 \%$ of indi- viduals over the age of 50, compared to $59 \%$ of those over the age of 55 in low-to-middle-income countries. ${ }^{4)}$ Thus, T2DM is a leading health concern in the aging population.

While aging is known to cause muscle weakness, aging combined with T2DM is a high-risk factor for the development of serious skeletal muscle deterioration. ${ }^{5,6)}$ Previous clinical studies on T2DM patients reported an accelerated loss of skeletal muscle mass in older adults with T2DM compared to non-diabetic older 
adults. $^{7-9)}$ Recent studies have suggested that the accelerated decrease in muscle mass and strength is associated with insulin resistance and diabetes complications; ${ }^{10,11)}$ however, the underlying mechanisms of these associations remain unclear. The loss of skeletal muscle mass and increased insulin resistance in older adults result in defective muscle function that may influence their strength, frailty, and even quality of life.

In older adults, T2DM is a crucial cause of mitochondrial dysfunction due to insulin resistance; moreover, resistance may contribute to a reduction in insulin-stimulated muscle glucose metabolism. ${ }^{12)}$ Hyperinsulinemia in the skeletal muscle of older adults reduces the anabolic protein response to insulin. ${ }^{13)}$ A study of single muscle fibers in a rat diabetes model did not observe a significant reduction in the contractile properties in the muscles of rats with diabetes. ${ }^{14)}$ The reduction in contractile force in the muscles of rats with diabetes was also shown to occur in a fiber-type dependent manner, with profound wasting in fast-twitch but not slowtwitch fibers. $^{15)}$

Berria et al. ${ }^{16)}$ reported that insulin-resistant skeletal muscle had increased collagen content, which is a precursor to defects in mitochondrial function and is related to abnormalities in the extracellular matrix (ECM). Since ECM in skeletal muscle contributes to a passive component of muscle force production, ${ }^{17)}$ the increased ECM content in aged T2DM muscles causes increased passive stiffness by elongation of skeletal muscle fibers. However, Pavan et al. ${ }^{18)}$ also showed that the aging of human skeletal muscle is associated with increased passive stiffness due to increased ECM stiffness mainly caused by collagen accumulation. Therefore, the underlying mechanisms by which the passive stiffness increases in aged human skeletal muscle with T2DM remain unclear.

There is a lack of studies quantifying the mechanical properties of human skeletal muscle fibers with diabetes. Thus, this study measured the active and passive components of the mechanical properties of skeletal muscle fibers obtained from older adults with T2DM and non-diabetic older adults to characterize the poor muscle quality of aged skeletal muscle with T2DM.

\section{MATERIALS AND METHODS}

\section{Sample Collection and Participants}

We collected two sets of skeletal muscle samples from older adults; namely, those with type 2 diabetes mellitus (T2DM, $>65$ years of age) and older adult control subjects (non-DM, > 65 years of age) who underwent elective orthopedic surgery at Seoul National University Bundang Hospital. Samples were removed from the gluteus maximus of six older adult patients with T2DM ( $81 \pm 3$ years of age) and seven control older adult subjects ( $73 \pm 2$ years of age).
We enrolled T2DM patients who were diagnosed with diabetes based on the American Diabetes Association (ADA) criteria [14]. Two of the six T2DM patients took metformin. The control group was enrolled based on the same ADA criteria. Patients diagnosed with muscle disease; peripheral arterial diseases; or chronic illnesses such as liver disease, renal failure, and cancer were excluded. Informed consent was obtained from all participants. This study was conducted according to the principles of the Declaration of Helsinki and was approved by the ethics committees of Seoul National University Bundang Hospital (No. B-0710/050-009).

\section{Muscle Fiber Preparation}

The bundles of muscle fibers from the collected samples were dissected and chemically permeabilized for the mechanical studies; the remainder of the muscle samples were snap-frozen in liquid nitrogen and stored at $-80^{\circ} \mathrm{C}$ for protein expression studies. The procedures for muscle fiber permeabilization have been described previously. ${ }^{19)}$ Briefly, the dissected muscle fiber bundles were soaked in a skinning solution (relaxing solution [RS] containing $1 \%(\mathrm{w} / \mathrm{v})$ Triton-X100 and protease inhibitors) and maintained for 24 hours in a slow shaker at $4^{\circ} \mathrm{C}$ to remove the sarcolemma and sarcoplasmic retinaculum. After permeabilization, the fiber bundles were washed thoroughly with RS for 12 hours in a slow shaker at $4^{\circ} \mathrm{C}$ and stored in $50 \%$ glycerol/ RS at $-20^{\circ} \mathrm{C}^{20}$ )

\section{Experimental Solutions}

This study used an RS, pre-activating solution (Pre-A), and maximal activating solution (AS). ${ }^{21)}$ All solutions contained N,N-bis(2-hydroxyethyl)-2-aminoethanesulfonic acid (BES), $40 \mathrm{mM}$; dithiothreitol (DDT), $1 \mathrm{mM}$; and creatine phosphate (PCr), $33 \mathrm{mM}$, and the ionic strength was adjusted to $180 \mathrm{mM}$ with K-proprionate, $\mathrm{pH} 7.0$ at $15^{\circ} \mathrm{C}^{22)}$ The RS, pre-A, and AS contained 6.86, 6.66, and $6.64 \mathrm{mM} \mathrm{MgCL} 2$, respectively. The Na-ATP compositions were 5.96, 5.98, and 6.23 mM; those for ethylene glycol-bis $(\beta$-aminoethyl ether)-N,N,N',N'-tetraacetic acid (EGTA) were 10, 1 , and 0 $\mathrm{mM}$; for Ca-EGTA, 0, 0, and $10 \mathrm{mM}$; and for K-propionate, 3.28, 30.44 , and $2.09 \mathrm{mM}$, respectively. All solutions had protease inhibitors (phenylmethylsulfonyl fluoride [PMSF], 0.5mM; leupeptin, $0.04 \mathrm{mM}$; and E64, $0.01 \mathrm{mM}$ ) to prevent protein degradation.

\section{Experimental Setup and Protocol}

Permeabilized single fibers were dissected and mounted using aluminum T clips between a length motor (ASI 322C; Aurora Scientific Inc., Aurora, Canada) and a force transducer element (ASI 403A, Aurora Scientific Inc.) in a skinned fiber apparatus (ASI 802D, Aurora Scientific Inc.) mounted on an inverted microscope (Nikon Inc., Tokyo, Japan). Sarcomere length (SL) was measured 
using a high-speed VSL camera and video-based SL software (ASI 901, Aurora Scientific Inc.). The experiments were performed at $15^{\circ} \mathrm{C}$. The fiber was set to slack length (the shortest length at which passive force first developed) with an average slack SL of $1.95 \mu \mathrm{m}$. The fiber width and depth (built-in prisms allowed for side views of the fibers and the measurement of depth) were measured at three points along the fiber, and the cross-sectional area $\left(\mathrm{CSA}, \mathrm{mm}^{2}\right.$ ) was calculated assuming an elliptical cross-section. The specific force was expressed as force per CSA $\left(\mathrm{mN} / \mathrm{mm}^{2}\right)$ and used for comparisons of force between groups.

A single fiber was placed in the RS (pCa 9.0) and then immersed in the pre-A (RS with a 10-fold lower EGTA concentration), followed by activation in AS (pCa 4.5). After maximal contraction was reached, the muscle fiber was quickly moved to the RS. To determine the passive tension-SL relationship, the fibers were stretched from slack SL to approximately $3.0 \mu \mathrm{m}$ of SL with elongation steps of $10 \%$ of fiber length. For this stretch-hold experiment, passive tension was measured at the end of the hold ( $30 \mathrm{sec}-$ onds after peak) to calculate the passive tension after the viscosity was removed. To determine the contributions of titin and ECM to the passive force, the titin anchors were extracted by incubating the muscle fibers in an RS containing $0.6 \mathrm{M} \mathrm{KCl}$ and then in an RS containing 1.0 M KI for 10 minutes each. ${ }^{23)}$ After the extraction process, the same stretch-hold experiment was performed to measure passive tension. The remaining tension, assumed to be the ECM-based passive force, was subtracted from the pre-extraction passive force to determine the titin-based passive force. After mechanical experiments, the single fibers were stored in an SDS sample buffer for gel electrophoresis and myosin isotyping.

\section{Analysis of Titin Protein Expression}

Titin isoform expression was determined using sodium dodecyl sulfate-polyacrylamide gel electrophoresis (SDS-PAGE) as described previously. ${ }^{24)}$ Briefly, frozen samples from each patient's gluteus maximus were solubilized in $8 \mathrm{M}$ urea buffer ( $8 \mathrm{M}$ urea, 2 M thiourea, 3\% SDS, $75 \mathrm{mM}$ DTT, $0.05 \mathrm{M}$ Tris- $\mathrm{HCl}, 0.03 \%$ bromophenol blue) and 50\% glycerol with leupeptin, E-64, and
PMSF inhibitors. The solubilized solutions were incubated for 10 minutes at $60^{\circ} \mathrm{C}$, centrifuged for 5 minutes at $12,580 \times \mathrm{g}$ to remove the particulate fraction, and the proteins were separated by electrophoresis. Then, the solubilized muscles were run on 1\% SDS-agarose gels, electrophoresed at $15 \mathrm{~mA}$ per gel for 3 hours and 20 minutes at $4^{\circ} \mathrm{C}$, as previously described. ${ }^{25)}$ The gels were stained with Coomassie Blue, scanned, and analyzed using One-D scan EX software (Scanalytics Inc., Rockville, MD, USA).

\section{Identification of Muscle Fiber Types}

SDS-PAGE was used to determine the myosin heavy chain (MHC) isoform composition of the muscle lysates and single fibers as previously described. ${ }^{25)}$ Preparation of muscle lysates was performed as described above for titin content analysis. The single fibers after mechanical experiments were stored in $30 \mu \mathrm{L}$ of SDS sample buffer containing $62.5 \mathrm{mM}$ Tris $\mathrm{pH} 6.8,2 \%$ SDS, $10 \%$ glycerol, $5 \%$ beta-mercaptoethanol, and $0.001 \%$ bromophenol blue at $-20^{\circ} \mathrm{C}$. The stacking gel contained a $4 \%$ acrylamide concentration ( $\mathrm{pH}$ 6.7), while the separating gel contained $6 \%$ acrylamide $(\mathrm{pH}$ $8.7)$ with $30 \%$ glycerol (v/v). The gels were run at a constant voltage of $140 \mathrm{~V}$ for 6 hours. The gels for muscle lysates were stained with Coomassie Blue and single fiber gels were silver-stained. $\mathrm{Hu}$ man or rat MHC standards were prepared from pooled muscle biopsy samples and run on each gel. The gels were scanned and analyzed using One-D scan EX software (Scanalytics Inc.). A representative MHC gel image is shown in Fig. 1.

\section{Statistical Analysis}

Data are presented as mean \pm standard error of the mean (SEM). GraphPad Prism 6 was used to calculate the statistical data. For statistical analysis, one-way analysis of variance (ANOVA) (comparison of fiber type distribution) and Student t-tests (comparison of $\mathrm{T} 2 \mathrm{DM}$ and non-DM) were used, as appropriate, with statistical significance defined as $\mathrm{p}<0.05$.

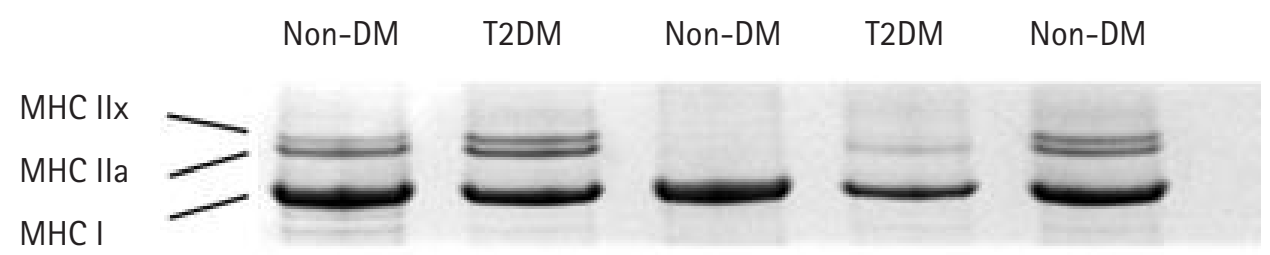

Fig. 1. Sodium dodecyl sulfate-polyacrylamide gel electrophoresis (SDS-PAGE) image of muscle lysates from older adults with type 2 diabetes mellitus (T2DM) and those without diabetes mellitus (non-DM). Each lane represents a muscle sample with bands from the top representing major histocompatibility complex (MHC) isoforms IIx, IIa, and I. 


\section{RESULTS}

\section{Fiber Type Distributions}

The ratio of the fiber type distribution for T2DM and non-DM subjects was determined by analyzing the MHC isoforms in muscle and single muscle fibers. The relative amounts of MHC I, IIa, and IIx were calculated to determine the distributions of fiber types I, IIa, and IIx, respectively. The results of the fiber type distribution are presented in Fig. 2, with a predominance of type I fibers ( $>80 \%$ ) observed in both T2DM and non-DM subjects $(\mathrm{p}<0.001)$. While non-DM muscles showed a greater type I fiber ratio ( $81 \%$ in T2DM and $88 \%$ in non-DM), no significant difference was found in the presence of T2DM (Fig. 2).

\section{Sizes and Active Contractile Properties of Single Muscle Fibers}

The average CSA of single type I muscle fibers in both T2DM and non-DM muscles are presented in Table 1. The CSA of the T2DM muscle was $19 \%$ smaller than that of the non-DM muscle $(3,577$ $\mu \mathrm{m}^{2}$ in T2DM and $4,391 \mu \mathrm{m}^{2}$ in non-DM), but the difference was not statistically significant. For mechanical experiments, only the results from type I fibers were analyzed because of the high pre-

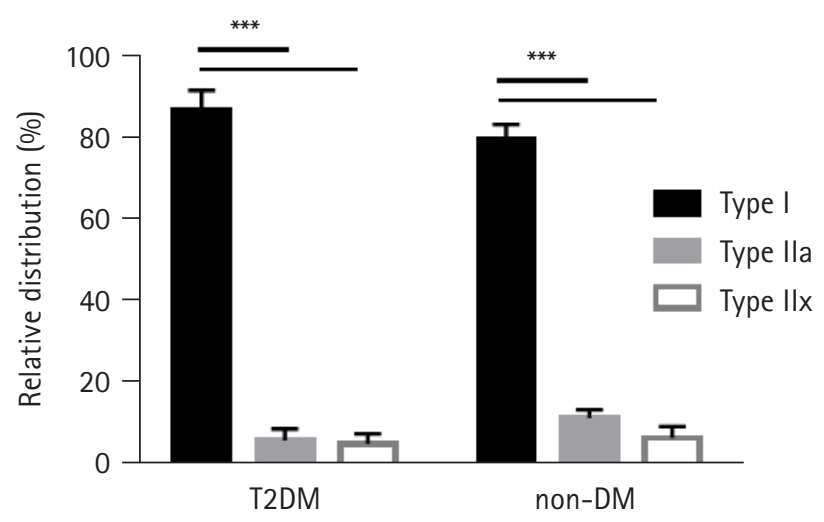

Fig. 2. Fiber type distribution as percentage of the total. The distribution of type I fibers is significantly greater in both type 2 diabetes mellitus (T2DM) and non-diabetes mellitus (non-DM) groups $\left({ }^{* * *} \mathrm{p}<0.0001\right)$. Type I fibers are predominant in both groups.

Table 1. CSA and maximal and specific forces of type I single muscle fibers in older adults with T2DM and non-DM

\begin{tabular}{lcc}
\hline & T2DM $(\mathrm{n}=9)$ & Non-DM $(\mathrm{n}=11)$ \\
\hline CSA $\left(\mu \mathrm{m}^{2}\right)$ & $3,577 \pm 368$ & $4,391 \pm 431$ \\
Maximal force $(\mu \mathrm{N})$ & $477 \pm 97$ & $668 \pm 59$ \\
Specific force $^{\mathrm{a}}\left(\mathrm{mN} / \mathrm{mm}^{2}\right)$ & $130 \pm 19$ & $155 \pm 07$ \\
\hline
\end{tabular}

Values are presented as mean \pm SEM.

CSA, cross-sectional area; T2DM, type 2 diabetes mellitus; DM, diabetes mellitus.

${ }^{\text {a) }}$ Specific force $=$ maximal force $/$ CSA. dominance of these fibers in both T2DM and non-DM muscles. The maximal force of T2DM muscle fibers $(477 \mu \mathrm{N})$ was approximately $70 \%$ of the maximal force of non-DM muscle fibers $(668 \mu \mathrm{N})$. The specific force was calculated as the maximal force divided by the fiber CSA and was 15\% smaller in the T2DM muscle $\left(130 \mathrm{mN} / \mathrm{mm}^{2}\right)$ compared to the non-DM muscle $(155 \mathrm{mN} /$ $\mathrm{mm}^{2}$ ) (Table 1). However, we observed no statistically significant differences in the contractile properties between T2DM and nonDM fibers.

\section{Passive Tensions of Single Muscle Fibers}

The SL-passive tension relationships of T2DM and non-DM type I single fibers are presented in Fig. 3. As expected, passive tension increased with stretch increments in both T2DM and non-DM muscles; however, T2DM muscle fibers showed a trend of larger increments in passive tension (SL 2.3-3.2 $\mu \mathrm{m}$ ) compared to fibers from non-DM subjects (circle in Fig. 3A). Significant differences in passive tension were found only for SLs of 2.9-3.2 $\mu \mathrm{m}$. Repeated measurement of the passive stretch-hold protocol after titin anchor extraction via incubation in $\mathrm{KCl} / \mathrm{CI}$ solution, titin-based passive tension, and ECM-based passive tension were determined for each fiber (see Methods section for more details). The relationships between SL and ECM-based passive tension (triangles in Fig. 3A) are also presented in Fig. 3A.

We found greater ECM-based passive tensions in T2DM muscle fibers (filled triangle in Fig. 3A) compared to those in non-DM muscle fibers (empty triangle in Fig. 3A). The differences between the two groups were statistically significant for SLs of 2.5-3.2 $\mu \mathrm{m}$. The ratio of titin-based and ECM-based passive tensions (titin/ ECM ratio) was calculated as the titin-based tension divided by ECM-based passive tension (Fig. 3B). The T2DM muscle fibers showed a significantly lower titin/ECM ratio than that in the nonDM group, which indicated that more ECM but less titin contributed to the total passive stiffness in the muscle fibers.

\section{Quantification of Titin Content}

The amounts of titin protein and MHC were determined by SDSPAGE and are presented in Table 2. While we observed a trend of greater titin and MHC content in T2DM muscles, the differences were not statistically significant. The amount of titin normalized to the MHC content in T2DM muscles was about $50 \%$ greater than that in non-DM muscles ( 0.15 for T2DM muscles and 0.1 for nonDM muscles), but no significant difference was observed between the two groups. 

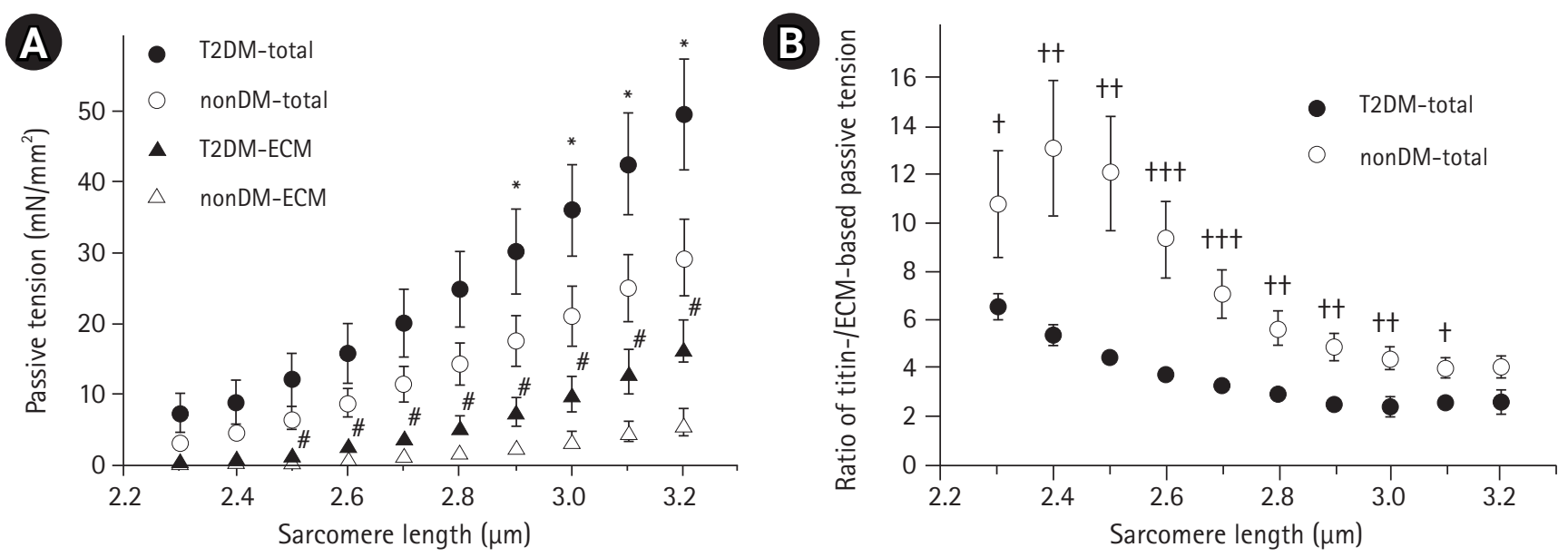

Fig. 3. (a) The relationships between sarcomere length and passive tension (total, circle) and extracellular matrix (ECM)-based passive tension (ECM, triangle) of single fibers from subjects with type 2 diabetes (T2DM) (filled symbol) and those without diabetes mellitus (non-DM) (empty symbol). The total and ECM-based passive tensions are consistently greater in T2DM muscles compared to those in non-DM groups. ${ }^{*}$ \# indicate comparisons of total and ECM-based passive tensions between the T2DM and non-DM groups, respectively ( $<<0.05)$. (b) The average ratio of titin-based and ECM-based passive tensions of single muscle fibers in the T2DM and non-DM groups. A lower ratio indicates a relatively higher contribution of ECM to the total passive stiffness of the muscle fibers. † indicates comparison between T2DM and non-DM groups $\left({ }^{+} \mathrm{p}<0.05,{ }^{++} \mathrm{p}<0.01,{ }^{+++} \mathrm{p}<0.001\right)$. Passive tensions were normalized according to the cross-sectional area, and values are shown as mean \pm SEM.

Table 2. Amounts of titin and MHC and the ratio of titin to MHC of muscle lysates from older adults with T2DM and non-DM subjects

\begin{tabular}{lcc}
\hline & T2DM $(\mathrm{n}=6)$ & Non-DM $(\mathrm{n}=7)$ \\
\hline Titin (a.u.) & $8,946 \pm 1920$ & $5,074 \pm 683$ \\
MHC (a.u.) & $55,390 \pm 5,459$ & $51,514 \pm 1,956$ \\
Titin/MHC & $0.15 \pm 0.03$ & $0.10 \pm 0.02$ \\
\hline
\end{tabular}

Values are presented as mean \pm SEM.

MHC, major histocompatibility complex; T2DM, type 2 diabetes mellitus; DM, diabetes mellitus.

\section{DISCUSSION}

Although abundant research has been conducted on diabetic skeletal muscles, ${ }^{26,27)}$ no studies have reported direct force measurements of human skeletal muscle fibers from patients with diabetes due to the difficulty of acquiring muscle fiber specimens from these patients. The reduction of muscle function and strength in diabetic patients and its acceleration with aging are well known. This study directly compared the active and passive mechanical properties of single skeletal muscle fibers from T2DM and nonDM subjects. To do so, we measured the size and active and passive forces of single muscle fibers using muscle mechanical experiments and analyzed the fiber type distributions and amounts of titin using a protein analysis technique in skeletal muscle samples from older adults with and without T2DM.

Consistent with previous studies on aged skeletal muscle, ${ }^{28,29)}$ we also confirmed the predominance of type I fibers ( $\mathrm{MHC}$ isoform I) in both the T2DM and non-DM groups (Fig. 2). Specifically, aging primarily affects type II fibers more than type I fibers; thus, aging is associated with a loss of muscular strength and power in older muscles. ${ }^{30)}$ Wang and Pessin ${ }^{31)}$ reported that type II fibers were more affected by cancer, diabetes, and chronic heart failure in addition to aging. While we expected to observe a greater percentage of type I fiber distribution in T2DM than in non-DM, our results showed the opposite: namely, a greater predominance in nonDM muscles. Sedentary lifestyles and inactivity due to lower extremity injuries (both T2DM and non-DM subjects underwent orthopedic surgery) may have affected the fiber type distributions.

Reduced muscle fiber size and active contractile force have been previously observed in diabetic and/or aged muscle fibers. Both aging and the presence of diabetes are risk factors for skeletal muscle dysfunction and loss of strength. ${ }^{5,6)}$ In this study, we confirmed reduced CSA and contractile force in human type I single muscle fibers with T2DM as compared to non-DM muscle (Table 1). The reduced specific tension in the T2DM muscle indicated impaired muscle quality due to diabetes in the muscles from older adults; however, the difference was not statistically significant. A study using a diabetic rat model reported more drastic changes in morphology and contractile function of single muscle fibers for type II muscle fibers than type I fibers in the presence of diabetes. ${ }^{32)}$ Since our study was limited to type I muscle fibers, further experiments with type II muscle fibers are required.

While we did not observe a significant association between muscle fiber size and active contractility of muscles in subjects in this study with T2DM, we did note a greater increase in passive 
tension in diabetic muscles compared to non-DM subjects within the physiological sarcomere working range (2.5-3.2 $\mu \mathrm{m})$ (Fig. 3A). Lim et al. ${ }^{29)}$ reported increased passive tension in aged type I single fibers compared to that in young control type I fibers in humans. Their finding indicates that some of our increased passive tension was associated with the aging process. We observed that the relationship between SL and ECM-based passive tension was significantly higher in the T2DM group than that in the non-DM group, which indicated that the ECM may be responsible for the increased passive tension in the muscle fibers from older adult subjects with T2DM (Fig. 3A). The ratio of titin/ECM-based passive tension was calculated to estimate the proportions of titin- and ECM-based passive tensions on the total passive stiffness. Regardless of SL and the presence of diabetes, titin-based passive tension was approximately 3- to 4-fold greater than ECM-based passive tension (ratios between 2 and 13) (Fig. 3B). However, this ratio was significantly lower in T2DM muscle fibers than that in nonDM fibers, which indicated a relatively higher ECM contribution to passive stiffness in T2DM fibers. An excessive accumulation of ECM inside and surrounding single fibers may reduce muscle function in older adults due to higher passive stiffness. ${ }^{18)}$ Previously, T2DM combined with muscle weakness due to aging reportedly resulted in ECM abnormality and reduced intermediate filament network. ${ }^{16)}$

Our findings support the idea that diabetes-induced additional accumulation of ECM in skeletal muscles due to increased insulin resistance may partially explain the passive component of functional disability in the skeletal muscles of subjects with T2DM. Further studies are needed to directly measure the ECM content in T2DM muscle and assess its association with passive stiffness. Additionally, the lack of information on insulin resistance factors in our participants provided limited evidence on the correlation between an additional increase in passive tension and T2DM. The extent to which the excessive accumulation of ECM is responsible for the T2DM muscle requires additional research.

\section{ACKNOWLEDGMENTS}

\section{CONFLICT OF INTEREST}

The researchers claim no conflicts of interest.

\section{FUNDING}

This research was supported by the Basic Science Research Program through the National Research Foundation of Korea (NRF) funded by the Ministry of Education (No. NRF-2016R1D1A1B03935518).

\section{AUTHOR CONTRIBUTION}

Conceptualization, EJL, JYL, HCJ, KHK; Data curation, EJL, JYL; Funding acquisition, JYL; Investigation, EJL, JYL; Methodology, EJL, JYL, HCJ, KHK, HYK; Project administration, JYL; Supervision, JYL, HCJ; Writing-original draft, EJL; Writing-review \& editing, EJL, JYL.

\section{REFERENCES}

1. Shaw JE, Sicree RA, Zimmet PZ. Global estimates of the prevalence of diabetes for 2010 and 2030. Diabetes Res Clin Pract 2010;87:4-14.

2. Whiting DR, Guariguata L, Weil C, Shaw J. IDF diabetes atlas: global estimates of the prevalence of diabetes for 2011 and 2030. Diabetes Res Clin Pract 2011;94:311-21.

3. Khan MA, Hashim MJ, King JK, Govender RD, Mustafa H, Al Kaabi J. Epidemiology of type 2 diabetes: global burden of disease and forecasted trends. J Epidemiol Glob Health 2020; 10:107-111.

4. Guariguata L, Whiting DR, Hambleton I, Beagley J, Linnenkamp U, Shaw JE. Global estimates of diabetes prevalence for 2013 and projections for 2035. Diabetes Res Clin Pract 2014; 103:137-49.

5. Park SW, Goodpaster BH, Strotmeyer ES, Kuller LH, Broudeau R, Kammerer C, et al. Accelerated loss of skeletal muscle strength in older adults with type 2 diabetes: the health, aging, and body composition study. Diabetes Care 2007;30:1507-12.

6. Jang HC. Diabetes and muscle dysfunction in older adults. Ann Geriatr Med Res 2019;23:160-4.

7. Park SW, Goodpaster BH, Lee JS, Kuller LH, Boudreau R, de Rekeneire N, et al. Excessive loss of skeletal muscle mass in older adults with type 2 diabetes. Diabetes Care 2009;32:1993-7.

8. Lee JS, Auyeung TW, Leung J, Kwok T, Leung PC, Woo J. The effect of diabetes mellitus on age-associated lean mass loss in 3153 older adults. Diabet Med 2010;27:1366-71.

9. Lee CG, Boyko EJ, Barrett-Connor E, Miljkovic I, Hoffman AR, Everson-Rose SA, et al. Insulin sensitizers may attenuate lean mass loss in older men with diabetes. Diabetes Care 2011;34: 2381-6.

10. Sinclair AJ, Abdelhafiz AH, Rodriguez-Manas L. Frailty and sarcopenia: newly emerging and high impact complications of diabetes. J Diabetes Complications 2017;31:1465-73.

11. Jang HC. Sarcopenia, frailty, and diabetes in older adults. Diabetes Metab J 2016;40:182-9.

12. Petersen KF, Befroy D, Dufour S, Dziura J, Ariyan C, Rothman DL, et al. Mitochondrial dysfunction in the elderly: possible role in insulin resistance. Science 2003;300:1140-2. 
13. Fujita S, Glynn EL, Timmerman KL, Rasmussen BB, Volpi E. Supraphysiological hyperinsulinaemia is necessary to stimulate skeletal muscle protein anabolism in older adults: evidence of a true age-related insulin resistance of muscle protein metabolism. Diabetologia 2009;52:1889-98.

14. Rodriguez-Reyes N, Rodriguez-Zayas AE, Javadov S, Frontera WR. Single muscle fiber contractile properties in diabetic RAT muscle. Muscle Nerve 2016;53:958-64.

15. Cotter M, Cameron NE, Lean DR, Robertson S. Effects of longterm streptozotocin diabetes on the contractile and histochemical properties of rat muscles. QJ Exp Physiol 1989;74:65-74.

16. Berria R, Wang L, Richardson DK, Finlayson J, Belfort R, Pratipanawatr $\mathrm{T}$, et al. Increased collagen content in insulin-resistant skeletal muscle. Am J Physiol Endocrinol Metab 2006;290: E560-5.

17. Marcucci L, Bondi M, Randazzo G, Reggiani C, Natali AN, Pavan PG. Fibre and extracellular matrix contributions to passive forces in human skeletal muscles: an experimental based constitutive law for numerical modelling of the passive element in the classical Hill-type three element model. PLoS One 2019;14: e0224232.

18. Pavan P, Monti E, Bondi M, Fan C, Stecco C, Narici M, et al. Alterations of extracellular matrix mechanical properties contribute to age-related functional impairment of human skeletal muscles. Int J Mol Sci 2020;21:3992.

19. Ottenheijm CA, Lawlor MW, Stienen GJ, Granzier H, Beggs $\mathrm{AH}$. Changes in cross-bridge cycling underlie muscle weakness in patients with tropomyosin 3-based myopathy. Hum Mol Genet 2011;20:2015-25.

20. Ogut O, Granzier H, Jin JP. Acidic and basic troponin T isoforms in mature fast-twitch skeletal muscle and effect on contractility. Am J Physiol 1999;276:C1162-70.

21. Fukuda N, Wu Y, Farman G, Irving TC, Granzier H. Titin-based modulation of active tension and interfilament lattice spacing in skinned rat cardiac muscle. Pflugers Arch 2005;449:449-57.

22. Fabiato A, Fabiato F. Calculator programs for computing the composition of the solutions containing multiple metals and ligands used for experiments in skinned muscle cells. J Physiol (Paris) 1979;75:463-505.

23. Wu Y, Cazorla O, Labeit D, Labeit S, Granzier H. Changes in titin and collagen underlie diastolic stiffness diversity of cardiac muscle. J Mol Cell Cardiol 2000;32:2151-62.

24. Hidalgo C, Saripalli C, Granzier HL. Effect of exercise training on post-translational and post-transcriptional regulation of titin stiffness in striated muscle of wild type and IG KO mice. Arch Biochem Biophys 2014;552-553:100-7.

25. Ottenheijm CA, Hidalgo C, Rost K, Gotthardt M, Granzier H. Altered contractility of skeletal muscle in mice deficient in titin's M-band region. J Mol Biol 2009;393:10-26.

26. D'Souza DM, Al-Sajee D, Hawke TJ. Diabetic myopathy: impact of diabetes mellitus on skeletal muscle progenitor cells. Front Physiol 2013;4:379.

27. Hernandez-Ochoa EO, Llanos P, Lanner JT. The underlying mechanisms of diabetic myopathy. J Diabetes Res 2017; 2017:7485738.

28. Talbot J, Maves L. Skeletal muscle fiber type: using insights from muscle developmental biology to dissect targets for susceptibility and resistance to muscle disease. Wiley Interdiscip Rev Dev Biol 2016;5:518-34.

29. Lim JY, Choi SJ, Widrick JJ, Phillips EM, Frontera WR. Passive force and viscoelastic properties of single fibers in human aging muscles. Eur J Appl Physiol 2019;119:2339-48.

30. Brunner F, Schmid A, Sheikhzadeh A, Nordin M, Yoon J, Frankel V. Effects of aging on type II muscle fibers: a systematic review of the literature. J Aging Phys Act 2007; 15:336-48.

31. Wang Y, Pessin JE. Mechanisms for fiber-type specificity of skeletal muscle atrophy. Curr Opin Clin Nutr Metab Care 2013;16: 243-50.

32. Huang L, Chen L, Qiu Y, Li S. Abnormalities in the fiber composition and contractility in diabetic skeletal muscles. Int J Clin Exp Med 2018;11:753-63. 